Kragujevac Journal of Mathematics

Volume 42(3) (2018), Pages 335-348.

\title{
APPROXIMATION RESULTS BY CERTAIN GENUINE OPERATORS OF INTEGRAL TYPE
}

\author{
VIJAY GUPTA ${ }^{1}$ AND DEEPIKA AGRAWAL ${ }^{2}$
}

\begin{abstract}
In the present article we propose genuine Lupaş-Beta operators of integral type. We establish quantitative asymptotic formula and a direct estimate in terms of second order modulus of continuity. Finally we consider the Bézier variant and obtain the rate of convergence for functions having derivatives of BV.
\end{abstract}

\section{INTRODUCTION}

Convergence estimates concerning linear positive operators is an active area of research among researchers in the last few decades. It is easier to construct new operators, but every time it is not straight forward to get their convergence estimates. In the year 1977 Pethe and Jain [8] proposed a general family of linear positive operators while generalizing Szász-Mirakjan operators. After three decades in the year 2007, Abel and Ivan [1] studied these operators in slightly different form and considered the following form of operators to establish complete asymptotic expansion. For $c=c_{n} \geq \beta, n=0,1, \ldots$, the operators discussed in [1] are defined as

$$
M_{n}^{c}(f, x)=\sum_{k=0}^{\infty} l_{n, k}^{c}(x) f\left(\frac{k}{n}\right), \quad x \geq 0,
$$

where $l_{n, k}^{c}(x)$ is given by

$$
l_{n, k}^{c}(x)=\left(\frac{c}{1+c}\right)^{n c x} \frac{(n c x)_{k}}{k ! \cdot(1+c)^{k}}
$$

Key words and phrases. Moments, factorial polynomials, Beta basis function, direct estimates, weighted modulus of continuity, $K$-functionals, bounded variation.

2010 Mathematics Subject Classification. Primary: 41A25. Secondary: 41A30.

Received: February 11, 2017.

Accepted: March 18, 2017. 
where $(a)_{k}$ is the rising factorial given by $(a)_{k}=a(a+1)(a+2) \cdots(a+k-1)$. These operators are well-defined, for all sufficiently large $n$, since the infinite sum in above form is convergent if $n>A / \log (1+c)$ (the case $c=1$ provides the Lupas operators [9]) provided that $|f(t)| \leq K e^{A t}(t \geq 0)$, that is $f \in E$. These operators as such can not be utilized to approximate integrable functions. In order to approximate integrable functions several hybrid Durrmeyer type operators have been considered in [3-7] with different weight function.

In the year 2008, Mihesan [10] proposed another form of general operator, which include some of the well known operators as special cases. The operators discussed in [10] for $x \in[0, \infty)$ is defined as

$$
M_{n}^{\alpha}(f, x)=\sum_{k=0}^{\infty} m_{n, k}(x, \alpha) f\left(\frac{k}{n}\right),
$$

where

$$
m_{n, k}(x, \alpha)=\frac{(\alpha)_{k}}{k !} \cdot \frac{\left(\frac{n x}{\alpha}\right)^{k}}{\left(1+\frac{n x}{\alpha}\right)^{\alpha+k}} .
$$

We observe here that the forms of the operators (1.1) and (1.2) are same, if we put $c=\alpha / n x$ in (1.1), we may get at once the operators $M_{n}^{\alpha}(f, x)$. We propose below the integral modification of the operators (1.2), by taking weights of Beta basis functions in the following way

$$
M_{n}^{\alpha}(f, x)=\sum_{k=1}^{\infty} m_{n, k}(x, \alpha) \int_{0}^{\infty} b_{n, k}(t) f(t) d t+\left(\frac{\alpha}{\alpha+n x}\right)^{\alpha} f(0),
$$

where $m_{n, k}(x, \alpha)$ is given by $(1.2)$ and

$$
b_{n, k}(t)=\frac{1}{B(n+1, k)} \cdot \frac{t^{k-1}}{(1+t)^{k+n+1}},
$$

where $B(m, n)$ being the Beta function. It is observed that these operators preserve linear functions. For different values of $\alpha$, one may get different special cases. Some of the special cases are indicated as below.

(i) In case if $\alpha \rightarrow \infty$, we obtain the Szász-Beta operators, which are defined by

$$
M_{n}^{\infty}(f, x)=\sum_{k=1}^{\infty} m_{n, k}(x, \infty) \int_{0}^{\infty} b_{n, k}(t) f(t) d t+e^{-n x} f(0)
$$

where

$$
m_{n, k}(x, \infty)=e^{-n x} \frac{(n x)^{k}}{k !}
$$

and $b_{n, k}(t)$ is as defined in (1.3). 
(ii) When $\alpha=n$, we obtain the Baskakov-Beta type operators, which for $x \geq 0$ are defined by

$$
M_{n}^{n}(f, x)=\sum_{k=1}^{\infty} m_{n, k}(x, n) \int_{0}^{\infty} b_{n, k}(t) f(t) d t+(1+x)^{-n} f(0),
$$

where

$$
m_{n, k}(x, n)=\frac{(n)_{k}}{k !} \cdot \frac{x^{k}}{(x+1)^{n+k}}=\left(\begin{array}{c}
n+k-1 \\
k
\end{array}\right) \frac{x^{k}}{(x+1)^{n+k}},
$$

and $b_{n, k}(t)$ is as defined in (1.3).

(iii) If we take $\alpha=n x$, the operators (1.3) will reduce to the Lupaş-Beta operators, defined as

$$
M_{n}^{n x}(f, x)=\sum_{k=1}^{\infty} m_{n, k}(x, n x) \int_{0}^{\infty} b_{n, k}(t) f(t) d t+2^{-n x} f(0),
$$

where

$$
m_{n, k}(x, n x)=2^{-n x} \frac{(n x)_{k}}{k ! 2^{k}}
$$

and $b_{n, k}(t)$ is as defined in (1.3).

Remark 1.1. When $\alpha=-n$, then the basis $m_{n, k}(x,-n)$ will reduce to the Bernstein basis function defined as

$$
m_{n, k}(x,-n)=\left(\begin{array}{l}
n \\
k
\end{array}\right) x^{k}(1-x)^{n-k} .
$$

As the operators (1.3) are defined on positive real axis, while the Bernstein basis function takes the valves in the interval $[0,1]$. So it is not often to consider such hybrid operators as this case.

In the present article, we establish quantitative asymptotic formula in terms of weighted modulus of continuity and a direct result in terms of second order modulus of continuity. Finally we consider the Bézier variant and obtain the rate of convergence.

\section{Moments}

In this section we find moments using the concept of Hypergeometric functions.

Lemma 2.1. For the monomial $M_{n}^{\alpha}\left(e_{r}, x\right), e_{r}(t)=t^{r}, r>0$, the $r$-th order moment satisfy the following relation

$$
M_{n}^{\alpha}\left(e_{r}, x\right)=\frac{x \cdot r !(n-r) !}{(n-1) !}{ }_{2} F_{1}\left(\alpha+1,1-r ; 2 ;-\frac{n x}{\alpha}\right) .
$$

Proof. Obviously using $\Gamma(r+k)=(r)_{k} . \Gamma r$, we have

$$
M_{n}^{\alpha}\left(e_{r}, x\right)=\sum_{k=1}^{\infty} \frac{(\alpha)_{k}}{k !} \cdot \frac{\left(\frac{n x}{\alpha}\right)^{k}}{\left(1+\frac{n x}{\alpha}\right)^{\alpha+k}} \cdot \frac{B(k+r, n-r+1)}{B(n+1, k)}
$$




$$
\begin{aligned}
& =\frac{(n-r) !}{n !} \cdot \frac{\Gamma r}{\left(1+\frac{n x}{\alpha}\right)^{\alpha}} \cdot \sum_{k=1}^{\infty} \frac{(\alpha)_{k}(r)_{k}}{k !(k-1) !} \frac{\left(\frac{n x}{\alpha}\right)^{k}}{\left(1+\frac{n x}{\alpha}\right)^{k}} \\
& =\frac{(n-r) !}{n !} \cdot \frac{\Gamma r}{\left(1+\frac{n x}{\alpha}\right)^{\alpha}} \cdot \sum_{k=0}^{\infty} \frac{\alpha r(\alpha+1)_{k}(r+1)_{k}}{k !(2)_{k}} \frac{\left(\frac{n x}{\alpha}\right)^{k+1}}{\left(1+\frac{n x}{\alpha}\right)^{k+1}} \\
& =\frac{\alpha \cdot r !(n-r) !}{n !\left(1+\frac{n x}{\alpha}\right)^{\alpha}}\left(\frac{n x}{\alpha+n x}\right){ }_{2} F_{1}\left(\alpha+1, r+1 ; 2 ; \frac{n x}{n x+\alpha}\right) .
\end{aligned}
$$

Using ${ }_{2} F_{1}(a, b ; c ; x)=(1-x)^{-a}{ }_{2} F_{1}\left(a, c-b ; c ; \frac{x}{x-1}\right)$, the result follows immediately.

This completes the proof of the lemma.

Remark 2.1. By simple computation from Lemma 2.1, the first few moments are given by

$$
\begin{aligned}
M_{n}^{\alpha}\left(e_{0}, x\right)= & 1 \\
M_{n}^{\alpha}\left(e_{1}, x\right)= & x, \\
M_{n}^{\alpha}\left(e_{2}, x\right)= & \frac{x[n x(\alpha+1)+2 \alpha]}{\alpha(n-1)}, \\
M_{n}^{\alpha}\left(e_{3}, x\right)= & \frac{x}{\alpha^{2}(n-1)(n-2)}\left[n^{2} x^{2}(\alpha+1)(\alpha+2)+6 n \alpha x(\alpha+1)+6 \alpha^{2}\right], \\
M_{n}^{\alpha}\left(e_{4}, x\right)= & \frac{24 x}{\alpha^{3}(n-1)(n-2)(n-3)}\left[n^{3} x^{3}(\alpha+1)(\alpha+2)(\alpha+3)\right. \\
& \left.+12 n^{2} x^{2} \alpha(\alpha+1)(\alpha+2)+36 n x \alpha^{2}(\alpha+1)+24 \alpha^{3}\right], \\
M_{n}^{\alpha}\left(e_{5}, x\right)= & \frac{x}{\alpha^{4}(n-1)(n-2)(n-3)(n-4)}\left[n^{4} x^{4}(\alpha+1)(\alpha+2)(\alpha+3)(\alpha+4)\right. \\
& +20 n^{3} x^{3} \alpha(\alpha+1)(\alpha+2)(\alpha+3)+120 n^{2} x^{2} \alpha^{2}(\alpha+1)(\alpha+2) \\
& \left.+240 n x \alpha^{3}(\alpha+1)+120 \alpha^{2}\right]
\end{aligned}
$$

and

$$
\begin{aligned}
M_{n}^{\alpha}\left(e_{6}, x\right)= & \frac{x}{\alpha^{5}(n-1)(n-2)(n-3)(n-4)(n-5)}\left[n^{5} x^{5}(\alpha+1)(\alpha+2)\right. \\
& \times(\alpha+3)(\alpha+4)(\alpha+5)+30 n^{4} x^{4} \alpha(\alpha+1)(\alpha+2)(\alpha+3)(\alpha+4) \\
& +300 n^{3} x^{3} \alpha^{2}(\alpha+1)(\alpha+2)(\alpha+3) \\
& \left.+1200 n^{2} x^{2} \alpha^{3}(\alpha+1)(\alpha+2)+1800 n x \alpha^{4}(\alpha+1)+720 \alpha^{5}\right] .
\end{aligned}
$$

Remark 2.2. If $\mu_{n, m}^{\alpha}(x)=M_{n}^{\alpha}\left((t-x)^{m}, x\right)$, then by simple computation using Remark 2.1, we have

$$
\mu_{n, 1}^{\alpha}(x)=0,
$$




$$
\begin{aligned}
\mu_{n, 2}^{\alpha}(x)= & \frac{x[x(n+\alpha)+2 \alpha]}{\alpha(n-1)}, \\
\mu_{n, 4}^{\alpha}(x)= & \frac{1}{\alpha^{3}(n-1)(n-2)(n-3)}\left[3 x ^ { 4 } \left(\alpha^{3}(n+6)+2 \alpha^{2} n(n+1)\right.\right. \\
& \left.+\alpha n^{2}(n+8)+2 n^{3}\right)+12 \alpha x^{3}\left(\alpha^{2}(n+1)+\alpha n(n+4)+2 n^{2}\right) \\
& \left.+12 \alpha^{2} x^{2}(\alpha(n+6)+3 n)+24 \alpha^{3} x\right]
\end{aligned}
$$

and

$$
\begin{aligned}
\mu_{n, 6}^{\alpha}(x)= & \frac{1}{\alpha^{5}(n-1)(n-2)(n-3)(n-4)(n-5)} \\
& \times\left[x ^ { 6 } \left[5 \alpha^{5}\left(3 n^{2}+86 n+120\right)+15 \alpha^{4} n\left(3 n^{2}+86 n+120\right)\right.\right. \\
& +5 \alpha^{3} n^{2}\left(9 n^{2}+284 n+480\right)+15 \alpha^{2} n^{3}\left(n^{2}+46 n+120\right) \\
& \left.+10 \alpha n^{4}(13 n+72)+120 n^{5}\right]+x^{5}\left[30 \alpha^{5}\left(3 n^{2}+8 n+120\right)\right. \\
& +60 \alpha^{4} n\left(3 n^{2}+86 n+120\right)+30 \alpha^{3} n^{2}\left(3 n^{2}+112 n+240\right) \\
& \left.+60 \alpha^{2} n^{3}(13 n+60)+720 \alpha n^{4}\right]+x^{4}\left[60 \alpha^{5}\left(3 n^{2}+86 n+120\right)\right. \\
& +180 \alpha^{4} n\left(n^{2}+33 n+60\right)+60 \alpha^{3} n^{2}(31 n+120) \\
& \left.+1800 \alpha^{2} n^{3}\right]+x^{3}\left[120 \alpha^{5}\left(n^{2}+33 n+60\right)+720 \alpha^{4} n(3 n+10)\right. \\
& \left.\left.+2400 \alpha^{3} n^{2}\right]+x^{2}\left[360 \alpha^{5}(3 n+10)+1800 \alpha^{4} n\right]+720 \alpha^{5} x\right] .
\end{aligned}
$$

\section{Direct Estimates}

Let $C_{x^{2}}[0, \infty)=C[0, \infty) \cap B_{x^{2}}[0, \infty)$, where $B_{x^{2}}[0, \infty)$ be the set of all functions $f$ defined on $\mathbb{R}^{+}$satisfying the condition $|f(x)| \leq M_{f}\left(1+x^{2}\right)$ with some constant $M_{f}$, depending only on $f$, but independent of $x$ by $C_{x^{2}}^{k}[0, \infty)$, we denote subspace of all continuous functions $f \in B_{x^{2}}[0, \infty)$ for which $\lim _{x \rightarrow \infty} \frac{f(x)}{1+x^{2}}$ is finite. The weighted modulus of continuity $\Omega(f, \delta)$ defined on infinite interval $\mathbb{R}^{+}$(see [2]) is defined as

$$
\boldsymbol{\Omega}(f, \delta)=\sup _{|h|<\delta, x \in \mathbb{R}^{+}} \frac{|f(x+h)-f(x)|}{\left(1+h^{2}\right)\left(1+x^{2}\right)}, \quad \text { for each } f \in C_{x^{2}}[0, \infty) .
$$

Now, some elementary properties of $\Omega(f, \delta)$ are collected in the following lemma.

Lemma 3.1. If $f \in C_{x^{2}}^{k}[0, \infty)$, then

(i) $\Omega(f, \delta)$ is monotonically increasing function of $\delta$, with $\delta \geq 0$;

(ii) for every $f \in C_{x^{2}}^{k}[0, \infty), \lim _{\delta \rightarrow 0} \Omega(f, \delta)=0$;

(iii) for each $\lambda>0$, we have $\Omega(f, \lambda \delta) \leq 2(1+\lambda)\left(1+\delta^{2}\right) \Omega(f, \delta)$.

We now estimate the following quantitative Voronovskaja-type asymptotic formula. 
Theorem 3.1. Let $f^{\prime \prime} \in C_{x^{2}}^{k}[0, \infty)$ and $x>0$ with the condition $\alpha=\alpha(n) \rightarrow \infty$ as $n \rightarrow \infty$. Also with this condition, we have $\lim _{n \rightarrow \infty} \frac{n}{\alpha(n)}=l$. Then, we have

$$
\begin{aligned}
& \left|M_{n}^{\alpha}(f, x)-f(x)-\left(\frac{x^{2} n+\left(x^{2}+2 x\right) \alpha}{\alpha(n-1)}\right) f^{\prime \prime}(x)\right| \\
\leq & C\left(1+x^{2}\right) \Omega\left(f^{\prime \prime}, n^{-1 / 2}\right)\left[\mu_{n, 2}^{\alpha}(x)+n^{2} \mu_{n, 6}^{\alpha}(x)\right],
\end{aligned}
$$

where $\mu_{n, 2}^{\alpha}(x)$ and $\mu_{n, 6}^{\alpha}(x)$ are given in Remark 2.2 and $C$ is certain constant less then 8 .

Proof. By Taylor's expansion, we have

$$
\begin{aligned}
M_{n}^{\alpha}(f, x)-f(x) & =M_{n}^{\alpha}((f(t)-f(x), x) \\
& =M_{n}^{\alpha}\left((t-x) f^{\prime}+\frac{(t-x)^{2}}{2} f^{\prime \prime}+h(t, x)(t-x)^{2}, x\right),
\end{aligned}
$$

where

$$
h(t, x):=\frac{f^{\prime \prime}(\eta)-f^{\prime \prime}(x)}{2}
$$

and $h$ is a continuous function which vanishes at 0 and $\eta$ lies between $x$ and $t$. Using Remark 2.2, we get

$$
\left|M_{n}^{\alpha}(f, x)-f(x)-\frac{f^{\prime \prime}(x)}{2}\left(\frac{x^{2} n+\left(x^{2}+2 x\right) \alpha}{\alpha(n-1)}\right)\right| \leq M_{n}^{\alpha}\left(|h(t, x)|(t-x)^{2}, x\right) .
$$

To estimate last inequality using Lemma 3.1 and the inequality $|\eta-x| \leq|t-x|$, we can write that

$$
|h(t, x)| \leq\left(1+(t-x)^{2}\right)\left(1+x^{2}\right)\left(1+\frac{|t-x|}{\delta}\right)\left(1+\delta^{2}\right) \Omega\left(f^{\prime \prime}, \delta\right) .
$$

Also,

$$
|h(t, x)| \leq \begin{cases}2\left(1+x^{2}\right)\left(1+\delta^{2}\right)^{2} \Omega\left(f^{\prime \prime}, \delta\right), & |t-x|<\delta \\ \left(1+(t-x)^{2}\right)\left(1+x^{2}\right)\left(1+\frac{|t-x|}{\delta}\right)\left(1+\delta^{2}\right) \Omega\left(f^{\prime \prime}, \delta\right), & |t-x| \geq \delta .\end{cases}
$$

Now choosing $\delta<1$, we have

$$
\begin{aligned}
|h(t, x)| & \leq 2\left(1+x^{2}\right)\left(1+\frac{(t-x)^{4}}{\delta^{4}}\right)\left(1+\delta^{2}\right)^{2} \Omega\left(f^{\prime \prime}, \delta\right) \\
& \leq 8\left(1+x^{2}\right)\left(1+\frac{(t-x)^{4}}{\delta^{4}}\right) \Omega\left(f^{\prime \prime}, \delta\right)
\end{aligned}
$$

Using Remark 2.2, we deduce that

$$
M_{n}^{\alpha}\left(|h(t, x)|(t-x)^{2}, x\right)=8\left(1+x^{2}\right) \Omega\left(f^{\prime \prime}, \delta\right)\left\{\mu_{n, 2}^{\alpha}(x)+\frac{1}{\delta^{4}} \mu_{n, 6}^{\alpha}(x)\right\} .
$$

Choosing $\delta=\frac{1}{\sqrt{n}}$ the result follows. 
Our next direct result is in terms of modulus of continuity, we first define some class. By $C_{B}[0, \infty)$, we mean the class of all real valued continuous and bounded functions $f$ on $[0, \infty)$. We denote the norm $\|f\|=\sup _{x \in[0, \infty)}|f(x)|$. For $f \in C_{B}[0, \infty)$ and $\delta>0$, the $\mathrm{m}$-th order modulus of continuity is defined as

$$
\omega_{m}(f, \delta)=\sup _{0 \leq h \leq \delta x \pm h \varphi(x) \in[0, \infty)} \sup _{h}\left|\Delta_{h}^{m} f(x)\right|,
$$

where $\Delta$ is the forward difference. In case $m=1$, we mean the usual modulus of continuity denoted by $\omega(f, \delta)$. The Peetre's K-functional is defined as

$$
K_{2}(f, \delta)=\inf _{g \in C_{B}^{2}[0, \infty)}\left\{\|f-g\|+\delta\left\|g^{\prime \prime}\right\|: g \in C_{B}^{2}[0, \infty)\right\}
$$

where

$$
C_{B}^{2}[0, \infty)=\left\{g \in C_{B}[0, \infty): g^{\prime}, g^{\prime \prime} \in C_{B}[0, \infty)\right\}
$$

Theorem 3.2. Let $f \in C_{B}[0, \infty)$ and $x \in[0, \infty)$, then

$$
\left|M_{n}^{\alpha}(f, x)-f(x)\right| \leq C \omega_{2}\left(f, \sqrt{\frac{x[x(n+\alpha)+2 \alpha]}{\alpha(n-1)}}\right),
$$

where $C$ is a positive constant.

Proof. Let $g \in C_{B}^{2}[0, \infty)$ and $x, t \in[0, \infty)$. By Taylor's formula, we have

$$
g(t)=g(x)+(t-x) g^{\prime}(x)+\int_{x}^{t}(t-u) g^{\prime \prime}(u) d u .
$$

Hence,

$$
\begin{aligned}
\left|M_{n}^{\alpha}(g, x)-g(x)\right| & =M_{n}^{\alpha}\left(\left|\int_{x}^{t}(t-u) g^{\prime \prime}(u) d u\right|, x\right) \\
& \leq M_{n}^{\alpha}\left((t-x)^{2}, x\right)\left\|g^{\prime \prime}\right\| \\
& =\frac{x[x(n+\alpha)+2 \alpha]}{\alpha(n-1)}\left\|g^{\prime \prime}\right\| .
\end{aligned}
$$

Now using operator (1.3), we have

$$
\begin{aligned}
\left|M_{n}^{\alpha}(f, x)\right| & \leq \sum_{k=1}^{\infty} m_{n, k}(x, \alpha) \int_{0}^{\infty} b_{n, k}(t)|f(t)| d t+\left(\frac{\alpha}{\alpha+n x}\right)^{\alpha}|f(0)| \\
& \leq\|f\| .
\end{aligned}
$$

Therefore

$$
\begin{aligned}
\left|M_{n}^{\alpha}(f, x)-f(x)\right| & \leq\left|M_{n}^{\alpha}(f-g, x)-(f-g)(x)\right|+\left|M_{n}^{\alpha}(g, x)-g(x)\right| \\
& \leq\|f-g\|+\frac{x[x(n+\alpha)+2 \alpha]}{\alpha(n-1)}\left\|g^{\prime \prime}\right\| .
\end{aligned}
$$

Lastly, taking infimum over all $g \in C_{B}^{2}[0, \infty)$, and using the inequality $K_{2}(f, \delta) \leq$ $C \omega_{2}(f, \sqrt{\delta}), \delta>0$, we get the desired result. 


\section{RATE of CONVERGENCE OF BÉZIER VARIANT}

For $\beta \geq 1$ the Bézier variant of the operators (1.3) is defined by

$$
M_{n}^{\alpha, \beta}(f, x)=\int_{0}^{\infty} K_{n, k}^{\beta}(x, t) f(t) d t,
$$

where the kernal $K_{n, k}^{\beta}(x, t)$ is given by

$$
K_{n, k}^{\beta}(x, t)=\sum_{k=1}^{\infty} Q_{n, k}^{(\beta)}(x, \alpha) b_{n, k}(t)+Q_{n, 0}^{(\beta)}(x, \alpha) \delta(t),
$$

$\delta(t)$ being the Dirac-delta function and $Q_{n, k}^{(\beta)}(x, \alpha)=\left[J_{n, k}(x, \alpha)\right]^{\beta}-\left[J_{n, k+1}(x, \alpha)\right]^{\beta}$ with $J_{n, k}(x, \alpha)=\sum_{j=k}^{\infty} m_{n, k}(x, \alpha)$. Clearly if $\beta=1$, then the operators $M_{n}^{\alpha, \beta}(f, x)$ reduce to the operators $M_{n}^{\alpha}(f, x)$.

Let $D_{\rho}\left(\mathbb{R}^{+}\right), \rho \geq 0$, denote the class of all absolutely continuous functions $f$ defined on $[0, \infty)$, having a derivative $f^{\prime}$ equivalent with a function of bounded variation on every finite sub-interval of $[0, \infty)$ and $|f(t)| \leq M t^{\rho}$. The functions $f \in D_{\rho}\left(\mathbb{R}^{+}\right)$ possess a representation

$$
f(x)=\int_{0}^{x} g(t) d t+f(0)
$$

where $g \in B V[0, \infty)$, i.e., $g$ is a function of bounded variation on every finite subinterval of $[0, \infty)$.

Lemma 4.1. For a fixed $x \in(0, \infty)$ with the condition $\alpha=\alpha(n) \rightarrow \infty$ as $n \rightarrow \infty$ and $\lim _{n \rightarrow \infty} \frac{n}{\alpha(n)}=l$, then for sufficiently large $n$, we have

(a) $\xi_{n, \alpha}^{\beta}(x, y)=\int_{0}^{y} K_{n, k}^{\beta}(x, t) d t \leq \frac{\beta x[x(l+1)+2]}{(n-1)} \frac{1}{(x-y)^{2}}, 0 \leq y<x$,

(b) $1-\xi_{n, \alpha}^{\beta}(x, z)=\int_{z}^{\infty} K_{n, k}^{\beta}(x, t) d t \leq \frac{\beta x[x(l+1)+2]}{(n-1)} \frac{1}{(z-x)^{2}}, x<z<\infty$.

Using Remark 2.2, the result follows immediately.

Theorem 4.1. Let $f \in D_{\rho}\left(\mathbb{R}^{+}\right)$. Then, for every $x \in(0, \infty)$ under the conditions of Lemma 4.1 for sufficiently large $n$, we have

$$
\begin{aligned}
& \left|M_{n}^{\alpha, \beta}(f, x)-f(x)\right| \\
\leq & \frac{\beta^{3 / 2}}{\beta+1}\left|f^{\prime}(x+)-f^{\prime}(x-)\right| \sqrt{\frac{x[x(l+1)+2]}{n-1}}+\frac{\beta x[x(l+1)+2]}{n-1} \sum_{k=1}^{[\sqrt{n}]} V_{x-x / k}^{x+x / k}\left(f_{x}^{\prime}\right) \\
& +\frac{x}{\sqrt{n}} V_{x-x / k}^{x+x / k}\left(f_{x}^{\prime}\right)+\frac{\beta[x(l+1)+2]}{x(n-1)}\left|f(2 x)-f(x)-x f^{\prime}(x+)\right| \\
& +\left|f^{\prime}(x+)\right| \sqrt{\frac{\beta x[x(l+1)+2]}{n-1}} \\
& +\frac{\beta C(n, \alpha, r, x)}{n^{r}}+\frac{|f(x)| \frac{\beta[x(l+1)+2]}{x}}{n-1}
\end{aligned}
$$


where $V_{a}^{b} f(x)$ denotes the total variation of $f(x)$ on $[a, b]$ and $f_{x}^{\prime}$ is defined by

$$
f_{x}^{\prime}(t)= \begin{cases}f^{\prime}(t)-f^{\prime}(x-), & 0 \leq t<x \\ 0, & t=x, \\ f^{\prime}(t)-f^{\prime}(x+), & x<t<\infty\end{cases}
$$

Proof. Since $M_{n}^{\alpha, \beta}(1, x)=1$, using (4.1), for every $x \in(0, \infty)$ we get

$$
\begin{aligned}
M_{n}^{\alpha, \beta}(f, x)-f(x) & =\int_{0}^{\infty} K_{n, k}^{\beta}(x, t)(f(t)-f(x)) d t \\
& =\int_{0}^{\infty} K_{n, k}^{\beta}(x, t) \int_{x}^{t} f^{\prime}(u) d u d t
\end{aligned}
$$

For any $f \in D_{\rho}\left(\mathbb{R}^{+}\right)$, from (4.2) we may write

$$
\begin{aligned}
f^{\prime}(u)= & f_{x}^{\prime}(u)+\frac{f^{\prime}(x+)+\beta f^{\prime}(x-)}{\beta+1}+\frac{f^{\prime}(x+)-f^{\prime}(x-)}{2}\left(\operatorname{sgn}(u-x)+\frac{\beta-1}{\beta+1}\right) \\
& +\delta_{x}(u)\left[f^{\prime}(u)-\frac{f^{\prime}(x+)+f^{\prime}(x-)}{2}\right],
\end{aligned}
$$

where

$$
\delta_{x}(u)= \begin{cases}1, & u=x \\ 0, & u \neq x\end{cases}
$$

From (4.3) and above expansion of $f^{\prime}(u)$, we get

$$
\begin{aligned}
M_{n}^{\alpha, \beta}(f, x)-f(x)= & \int_{0}^{\infty} K_{n, k}^{\beta}(x, t) \int_{x}^{t}\left[f_{x}^{\prime}(u)+\frac{f^{\prime}(x+)+\beta f^{\prime}(x-)}{\beta+1}\right. \\
& +\frac{f^{\prime}(x+)-f^{\prime}(x-)}{2} \cdot\left(\operatorname{sgn}(u-x)+\frac{\beta-1}{\beta+1}\right) \\
& \left.+\delta_{x}(u)\left[f^{\prime}(u)-\frac{f^{\prime}(x+)+f^{\prime}(x-)}{2}\right] d u\right] d t \\
:= & A_{n}^{\alpha, \beta}\left(f_{x}^{\prime}, x\right)+B_{n}^{\alpha, \beta}\left(f_{x}^{\prime}, x\right)+A_{1}+A_{2}+A_{3},
\end{aligned}
$$

where

$$
\begin{aligned}
A_{n}^{\alpha, \beta}\left(f_{x}^{\prime}, x\right) & =\int_{0}^{x}\left(\int_{x}^{t} f_{x}^{\prime}(u) d u\right) K_{n, k}^{\beta}(x, t) d t \\
B_{n}^{\alpha, \beta}\left(f_{x}^{\prime}, x\right) & =\int_{x}^{\infty}\left(\int_{x}^{t} f_{x}^{\prime}(u) d u\right) K_{n, k}^{\beta}(x, t) d t \\
A_{1} & =\int_{0}^{\infty}\left(\int_{x}^{t} \frac{f^{\prime}(x+)+\beta f^{\prime}(x-)}{\beta+1} d u\right) K_{n, k}^{\beta}(x, t) d t \\
A_{2} & =\int_{0}^{\infty} K_{n, k}^{\beta}(x, t)\left(\int_{x}^{t} \frac{f^{\prime}(x+)-f^{\prime}(x-)}{2}\left(\operatorname{sgn}(u-x)+\frac{\beta-1}{\beta+1}\right) d u\right) d t, \\
A_{3} & =\int_{0}^{\infty}\left(\int_{x}^{t}\left(f^{\prime}(u)-\frac{f^{\prime}(x+)+f^{\prime}(x-)}{2}\right) \delta_{x}(u) d u\right) K_{n, k}^{\beta}(x, t) d t .
\end{aligned}
$$


Using Remark 2.2, we get

$$
\begin{aligned}
A_{1} & =\frac{f^{\prime}(x+)+\beta f^{\prime}(x-)}{\beta+1} \int_{0}^{\infty}(t-x) K_{n, k}^{\beta}(x, t) d t \\
& =\frac{f^{\prime}(x+)+\beta f^{\prime}(x-)}{\beta+1} \cdot M_{n}^{\alpha, \beta}((t-x), x)=0 .
\end{aligned}
$$

Also using Remark 2.2, for sufficiently large $n$, we get

$$
\begin{aligned}
A_{2}= & \int_{0}^{\infty} K_{n, k}^{\beta}(x, t)\left(\int_{x}^{t} \frac{f^{\prime}(x+)-f^{\prime}(x-)}{2}\left(\operatorname{sgn}(u-x)+\frac{\beta-1}{\beta+1}\right) d u\right) d t \\
= & \frac{f^{\prime}(x+)-f^{\prime}(x-)}{2}\left[-\int_{0}^{x}\left(\int_{t}^{x}\left(\operatorname{sgn}(u-x)+\frac{\beta-1}{\beta+1}\right) d u\right) K_{n, k}^{\beta}(x, t) d t\right. \\
& \left.+\int_{x}^{\infty}\left(\int_{x}^{t}\left(\operatorname{sgn}(u-x)+\frac{\beta-1}{\beta+1}\right) d u\right) K_{n, k}^{\beta}(x, t) d t\right] \\
\leq & \frac{\beta}{\beta+1}\left|f^{\prime}(x+)-f^{\prime}(x-)\right| \int_{0}^{\infty}|t-x| K_{n, k}^{\beta}(x, t) d t \\
= & \frac{\beta}{\beta+1}\left|f^{\prime}(x+)-f^{\prime}(x-)\right| M_{n}^{\alpha, \beta}(|t-x|, x) \\
= & \frac{\beta^{3 / 2}}{\beta+1}\left|f^{\prime}(x+)-f^{\prime}(x-)\right| \sqrt{\frac{x[x(l+1)+2]}{n-1}} .
\end{aligned}
$$

Obviously, $A_{3}=0$. Thus our problem is reduced to calculate the estimates of the terms $A_{n}^{\alpha, \beta}\left(f_{x}^{\prime}, x\right)$ and $B_{n}^{\alpha, \beta}\left(f_{x}^{\prime}, x\right)$. Since $\int_{a}^{b} d_{t} \xi_{n, k}^{\beta}(x, t) \leq 1$ for all $[a, b] \subseteq[0, \infty)$, using integration by parts and applying Lemma 4.1 with $y=x-x / \sqrt{n}$, we have

$$
\begin{aligned}
\left|A_{n}^{\alpha, \beta}\left(f_{x}^{\prime}, x\right)\right| & =\left|\int_{0}^{x}\left(\int_{x}^{t} f_{x}^{\prime}(u) d u\right) d_{t} \xi_{n, k}^{\beta}(x, t)\right|=\left|\int_{0}^{x} \xi_{n, k}^{\beta}(x, t) f_{x}^{\prime}(t) d t\right| \\
& \leq \int_{0}^{y}\left|f_{x}^{\prime}(t)\right|\left|\xi_{n, k}^{\beta}(x, t)\right| d t+\int_{y}^{x}\left|f_{x}^{\prime}(t)\right|\left|\xi_{n, k}^{\beta}(x, t)\right| d t \\
& \leq \frac{\beta x[x(l+1)+2]}{n-1} \int_{0}^{y} V_{t}^{x}\left(f_{x}^{\prime}\right)(x-t)^{-2} d t+\int_{y}^{x} V_{t}^{x}\left(f_{x}^{\prime}\right) d t \\
& \leq \frac{\beta x[x(l+1)+2]}{n-1} \int_{0}^{y} V_{t}^{x}\left(f_{x}^{\prime}\right)(x-t)^{-2} d t+\frac{x}{\sqrt{n}} V_{x-x / \sqrt{n}}^{x}\left(f_{x}^{\prime}\right) \\
& =\frac{\beta x[x(l+1)+2]}{n-1} \int_{0}^{x-x / \sqrt{n}} V_{t}^{x}\left(f_{x}^{\prime}\right)(x-t)^{-2} d t+\frac{x}{\sqrt{n}} V_{x-x / \sqrt{n}}^{x}\left(f_{x}^{\prime}\right) \\
& \leq \frac{\beta x[x(l+1)+2]}{n-1} \sum_{k=1}^{[\sqrt{n}} V_{x-x / k}^{x}\left(f_{x}^{\prime}\right)+\frac{x}{\sqrt{n}} V_{x-x / \sqrt{n}}^{x}\left(f_{x}^{\prime}\right) .
\end{aligned}
$$


Finally, using integration by parts in $B_{n}^{\alpha, \beta}\left(f_{x}^{\prime}, x\right)$ and applying Lemma 4.1 and CauchySchwarz inequality, we have

$$
\begin{aligned}
\left|B_{n}^{\alpha, \beta}\left(f_{x}^{\prime}, x\right)\right| \leq & M\left|\int_{2 x}^{\infty}\left(\int_{x}^{t} f_{x}^{\prime}(u) d u\right) d t K_{n, k}^{\beta}(x, t)\right| \\
& +\left|\int_{x}^{2 x}\left(\int_{x}^{t} f_{x}^{\prime}(u) d u\right) d_{t}\left(1-\xi_{n, \alpha}^{\beta}(x, t)\right)\right| \\
\leq & \left|\int_{2 x}^{\infty}(f(t)-f(x)) K_{n, k}^{\beta}(x, t)\right| \\
& +\left|f^{\prime}(x+)\right|\left|\int_{2 x}^{\infty}(t-x) K_{n, k}^{\beta}(x, t) d t\right| \\
& +\left|\int_{x}^{2 x} f_{x}^{\prime}(u) d u\right|\left|1-\xi_{n, \alpha}^{\beta}(x, 2 x)\right| \\
& +\left|\int_{x}^{2 x} f_{x}^{\prime}(t)\left(1-\xi_{n, \alpha}^{\beta}(x, t)\right) d t\right| \\
\leq & \int_{2 x}^{\infty} f(t) K_{n, k}^{\beta}(x, t)|+| f(x)|| \int_{2 x}^{\infty} K_{n, k}^{\beta}(x, t) \mid \\
& +\left|f^{\prime}(x+)\right|\left(\int_{2 x}^{\infty}(t-x)^{2} K_{n, k}^{\beta}(x, t) d t\right)^{1 / 2} \\
& +\frac{\beta x[x(l+1)+2]}{n-1}\left|\int_{x}^{2 x}\left(\left(f^{\prime}(u)\right)-f^{\prime}(x+)\right) d u\right| \\
& +\left|\int_{x}^{x+x / \sqrt{n}} f_{x}^{\prime}(t) d t\right| \\
& +\frac{\beta x[x(l+1)+2]}{n-1}\left|\int_{x+x / \sqrt{n}}^{2 x}(t-x)^{-2} f_{x}^{\prime}(t) d t\right| \\
& \mid
\end{aligned}
$$

We see that there exists an integer $r, 2 r \geq \rho$, such that $f(t)=O\left(t^{2 r}\right)$, as $t \rightarrow \infty$. Now proceeding in a manner similar to the estimate of $A_{n}^{\alpha, \beta}\left(f_{x}^{\prime}, x\right)$, on substituting $t=x+\frac{x}{u}$ and in the last step using the Remark 2.2 and Lemma 4.1, we get

$$
\begin{aligned}
\left|B_{n}^{\alpha, \beta}\left(f_{x}^{\prime}, x\right)\right| \leq & M\left|\int_{2 x}^{\infty} t^{2 r} K_{n, k}^{\beta}(x, t) d t\right|+|f(x)| \int_{2 x}^{\infty} K_{n, k}^{\beta}(x, t) d t \\
& +\left|f^{\prime}(x+)\right| \sqrt{\frac{\beta x[x(l+1)+2]}{n-1}} \\
& +\frac{\beta[x(l+1)+2]}{x(n-1)}\left|f(2 x)-f(x)-x f^{\prime}(x+)\right| \\
& +\frac{x}{\sqrt{n}} V_{x}^{x+x / \sqrt{n}}\left(f_{x}^{\prime}\right)
\end{aligned}
$$




$$
\begin{aligned}
& \quad+\frac{\beta x[x(l+1)+2]}{n-1} \sum_{k=1}^{[\sqrt{n}]} V_{x}^{x+x / \sqrt{n}}\left(f_{x}^{\prime}\right) \\
& \leq \frac{\beta C(n, \alpha, r, x)}{n^{r}}+\frac{|f(x)| \frac{\beta[x(l+1)+2]}{x} \frac{(n-1)}{n-1}}{+} \\
& +\left|f^{\prime}(x+)\right| \sqrt{\frac{\beta x[x(l+1)+2]}{n-1}} \\
& +\frac{\beta[x(l+1)+2]}{x(n-1)}\left|f(2 x)-f(x)-x f^{\prime}(x+)\right| \\
& +\frac{x}{\sqrt{n}} V_{x}^{x+x / \sqrt{n}}\left(f_{x}^{\prime}\right) \\
& +\frac{\beta x[x(l+1)+2]}{n-1} \sum_{k=1}^{[\sqrt{n}]} V_{x}^{x+x / k}\left(f_{x}^{\prime}\right) .
\end{aligned}
$$

Collecting the estimates $A_{1}-A_{3}$ and $A_{n}^{\alpha, \beta}\left(f_{x}^{\prime}, x\right)$ and $B_{n}^{\alpha, \beta}\left(f_{x}^{\prime}, x\right)$, we get the required result. This completes the proof.

\section{Graphical Representation}

The convergence of the operators $M_{n}^{\alpha}(f, x)$ for the functions $f(x)=x^{2}+2 x+7$ (Figure 1 and Figure 2) and $f(x)=x^{3}+4 x^{2}+3 x+8$ (Figure 3 and Figure 4) are indicated below.

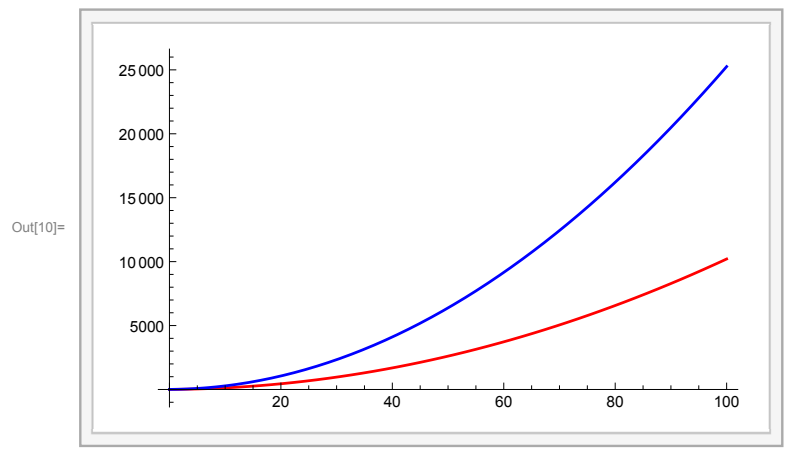

Figure 1. Convergence of the operators $M_{n}^{\alpha}(f, x)$ for the functions $f(x)=x^{2}+2 x+7, \alpha=1, n=5$

From the above figures, we conclude that for large values of $\alpha$ and $n, M_{n}^{\alpha}(f, x)$ converges more rapidly to the function $f(x)$. 


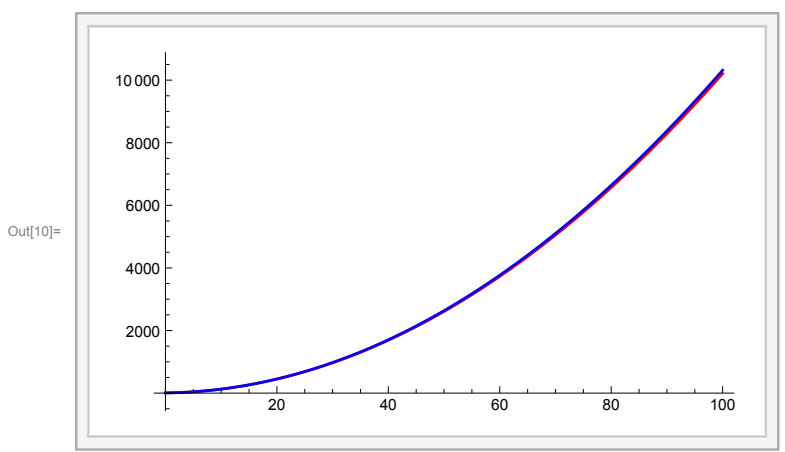

Figure 2. Convergence of the operators $M_{n}^{\alpha}(f, x)$ for the functions $f(x)=x^{2}+2 x+7, \alpha=100, n=1000$

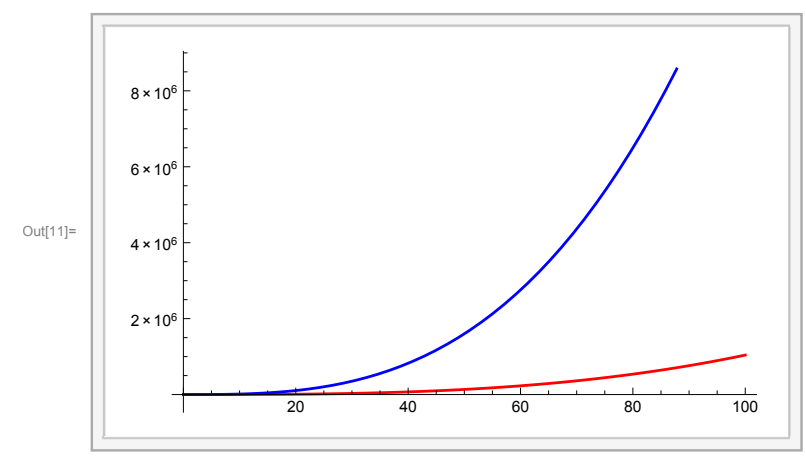

Figure 3. Convergence of the operators $M_{n}^{\alpha}(f, x)$ for the functions $f(x)=x^{3}+4 x^{2}+3 x+8, \alpha=1, n=5$

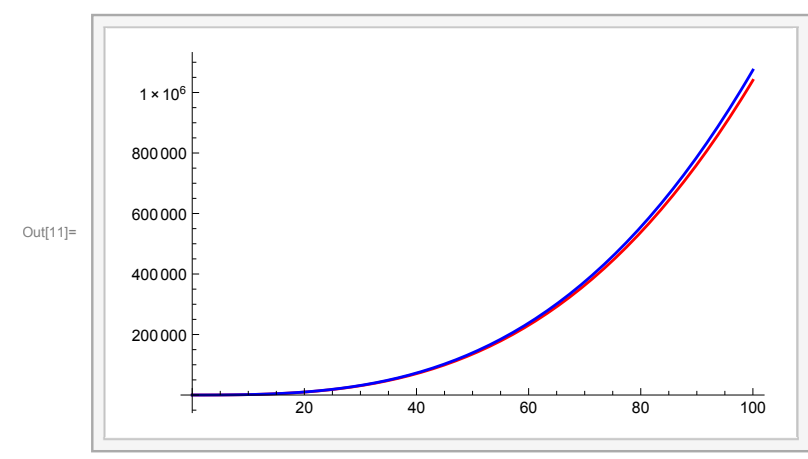

Figure 4. Convergence of the operators $M_{n}^{\alpha}(f, x)$ for the functions $f(x)=x^{3}+4 x^{2}+3 x+8, \alpha=100, n=1000$

Acknowledgements. The authors are thankful to the reviewer(s) for valuable suggestions. 


\section{REFERENCES}

[1] U. Abel and M. Ivan, On a generalization of an approximation operator defined by A. Lupaş, Gen. Math. 15(1) (2007), 21-34.

[2] T. Acar, A. Aral and I. Rasa, The new forms of Voronovskaja's theorem in weighted spaces, Positivity 20 (2016), 25-40.

[3] V. Gupta, Direct estimates for a new general family of Durrmeyer type operators, Boll. Unione Mat. Ital. 7 (2015), 279-288.

[4] Z. Finta and V. Gupta, Some results on modified Szász-Mirakjan operators, J. Math. Anal. Appl. 327(2) (2007), 1284-1296.

[5] V. Gupta and R. P. Agarwal, Convergence Estimates in Approximation Theory, Springer, Cham, 2014.

[6] V. Gupta, Th. M. Rassias and R. Yadav, Approximation by Lupaş-Beta integral operators, Appl. Math. Comput. 236 (2014), 19-26.

[7] V. Gupta and R. Yadav, On approximation of certain integral operators approximation of certain integral operators, Acta Math. Vietnam. 39(2) (2014), 193-203.

[8] G. C. Jain and S. Pethe, On the generalizations of Bernstein and Szász-Mirakjan operators, Nanta Math. 10 (1977), 185-193.

[9] A. Lupas, The approximation by means of some linear positive operators, in: Approximation Theory (Proceedings of the International Dortmund Meeting IDoMAT 95, held in Witten, Germany, March 13-17, 1995), M. W. Muller, M. Felten and D. H. Mache, eds. (Mathematical research 86) Akademie Verlag, Berlin 1995, pp. 201-229.

[10] V. Miheşan, Gamma approximating operators, Creat. Math. Inform. 17(3) (2008), 466-472.

${ }^{1}$ Department of Mathematics,

Netaji Subhas Institute of Technology,

Sector 3 DWARKA, NeW Delhi 110078, IndiA

E-mail address: vijaygupta2001@hotmail.com

${ }^{2}$ Department of Mathematics

Netaji Subhas Institute of Technology

SeCtor 3 DWARKA, New Delhi 110078, IndiA

E-mail address: deepikaagrawal304@gmail.com 